\title{
A simple construction of certain diffusion processes
}

\author{
By \\ John LAMPERTI ${ }^{1)}$ \\ (Communicated by Prof. K. Itô) \\ (Received Aug. 24, 1964)
}

\section{The construction}

The purpose of this note is to discuss what I believe to be the simplest rigorous approach to one-dimensional diffusion theory. The method used is not really new; the idea appears in some of the older physically-oriented literature, although usually in a mathematically incomplete form, and the same basic approach has been taken recently by McKean [10] in the case of Bessel processes. The possibilities of the method do not seem to have been widely appreciated, however. Although the class of diffusions which can easily be derived in this way is limited, the method does in these cases offer easy proofs of some rather deep results on the nature of sample functions.

We will obtain other diffusions from the standard Wiener (Brownian motion) process, which is here taken for granted, by a method quite reminiscent of $\mathrm{K}$. Itô's approach via stochastic differential equations [9] (see also [3], chapter 9). However, the concept of a stochastic integral will not be needed. Let $m(y)$ be a real function which satisfies a Lipschitz condition of order 1 on the whole line, and consider the integral equation

$$
y(t)=a+\int_{0}^{t} m[y(\tau)] d \tau+x(t) .
$$

1) This work was supported in part by the National Science Foundation. 
It is well known that there is a unique continuous solution $y(t)$ for each continuous function $x(t)$ and constant $a$, and that the mapping $M_{a}$ taking $x$ into $y$ is continuous in the topology of uniform convergence on finite intervals. As a result, if $x(t)$ is considered to be a sample function of the Wiener process, $y(t)$ will also be a path of a stochastic process, and since the Wiener paths are almost surely continuous the same will hold for $\{y(t)\}$.

To make this more precise, let $W$ denote the Wiener measure on the Borel sets of, say, $C_{(0,1)}$. Since $M_{a}$ is continuous, it is possible to define a new probability measure $P_{a}$ by putting $P_{a}(A)$ $=W\left(M_{a}^{-1} A\right)$ for any Borel set $A$; we can then consider $\{y(t)\}$ as a random function defined on the sample space $\left(C_{(0,1)}, P_{a}\right)$. Since almost all Wiener paths start at 0 we clearly have $P_{a}\{y(0)=a\}=1$.

It is not hard to see that $\{y(t)\}$ is a Markov process with stationary transition probabilities. If (1) is rewritten in the form

$$
y(t+s)=y(t)+\int_{t}^{t+s} m[y(\tau)] d \tau+[x(t+s)-x(t)],
$$

we see that $y(t+s)$ depends only on $y(t)$ and increments $x(t+\tau)-x(t)$, $\tau>0$. But $y(t)$ depends on $x(\tau)$ for $\tau \leq t$, and these values are independent of the increments above. It follows that the conditional distributions of $y(t+s)$ given $y(t)$ only and given $\{y(\tau), \tau \leq t\}$ are the same, which is a form of the Markov property. It is also clear from (2), because of the stationarity of the increments of Wiener processes, that the conditional distribution of $y(t+s)$ given $a=y(t)$ is equal (a.s.) to the distribution of $y(s)$ computed with initial state $a$. Thus our claim can be quite easily substantiated.

There is a simple step by which the construction can be considerably generalized : let $f$ be a continuous, strictly increasing function from $(-\infty, \infty)$ into itself, and let $z(t)=f(y(t))$. Obviously the Markov property, stationary transition probabilities, and continuity of paths enjoyed by the process $\{y(t)\}$ are still present in $\{z(t)\}$; in other words, $\{z(t)\}$ is also a diffusion process. It follows from a theorem of Dynkin and Yushkevich [5] (or [8]) that the strong Markov property is also present, but we shall not discuss that in detail or make use of it here. 


\section{Generator of $\{y(t)\}$}

That is all there is to the construction, but it remains to be seen which processes can be so obtained. In this section we will characterize the process $\{y(t)\}$ resulting from the first stage of the construction by finding the infinitesimal generator of an associated semi-group of transformations. Let $C_{0}$ be the space of continuous real-valued functions having limits at $\pm \infty$, aud define

$$
\left(\boldsymbol{T}_{t} u\right)(a)=E[u(y(t)) \mid y(0)=a]
$$

for functions $u \in C_{0}$. We will first show that $\boldsymbol{T}_{t}$ is a contraction semi-group of operators from $C_{0}$ into itself; the only part of this assertion which is not obvious is the continuity of $\boldsymbol{T}_{t} u$ at $\pm \infty$. This is very easily seen if the function $m(y)$ is bounded, for then $y(t)$ differs from Brownian motion by at most a fixed quantity (for each $t)$.

In case $m(y)$ is not bounded, we will prove continuity using an inequality also needed later, which we now derive. From (1) follows

$$
|y(t)-a| \leq|x(t)|+t|m(a)|+K \int_{0}^{t}|y(\tau)-a| d \tau,
$$

where $K$ is the constant in the Lipschitz condition satisfied by $m$. If $t>0$, and $t^{*}$ is a point in $[0, t]$ at which $|y(\sigma)-a|$ attains its maximum for that interval, then

$$
\left|y\left(t^{*}\right)-a\right| \leq\left|x\left(t^{*}\right)\right|+t^{*}|m(a)|+K t^{*}\left|y\left(t^{*}\right)-a\right| \text {. }
$$

It is an easy consequence that for values of $t$ such that $1-K t>\frac{1}{2}$

$$
\max _{\tau \leq t}|y(\tau)-a| \leq 2 t|m(a)|+2 \max _{\tau \leq t}|x(\tau)| .
$$

Now suppose $u \in C_{0}$, and $u(\infty)=b$, say. To prove that $\boldsymbol{T}_{t} u(\infty)$ $=b$ also, it suffices to show that

$$
\lim _{a \rightarrow \infty} \operatorname{Pr}(y(t) \leq A \mid y(0)=a)=0
$$

for every finite $A$; furthermore, it is enough to prove it for all small enough $t$. Now because of the Lipschitz condition, $|m(a)| \leq$ $|m(0)|+K|a|$. Then from (4) we have for $1-2 K t>0$ that 


$$
\begin{gathered}
\operatorname{Pr}(y(t) \leq A \mid y(0)=a) \leq \operatorname{Pr}(|y(t)-a| \geq a-A \mid y(0)=a) \\
\leq \operatorname{Pr}\left(2 \max _{\tau \leq t}|x(\tau)| \geq a(1-2 t K)+B\right)
\end{gathered}
$$

where $B$ is constant. Since $\max _{\tau \leq t}|x(\tau)|$ has a distribution which does not depend on $a$, we obviously obtain 0 as the limit when $a \rightarrow \infty$, and hence (5). Continuity at $-\infty$ is proved similarly.

Let $\boldsymbol{A}$ denote the (strong) infinitesimal generator of $\boldsymbol{T}_{t}$. We will prove

Theorem 1. The generator of $\left\{\boldsymbol{T}_{t}\right\}$ is given by

$$
(\boldsymbol{A} u)(a)=m(a) u^{\prime}(a)+\frac{1}{2} u^{\prime \prime}(a) ;
$$

the domain of $\boldsymbol{A}$ consists of exactly those functions $u$ which have a continuous second derivative and for which the right hand side of (6) tends to 0 as $a \rightarrow \pm \infty$.

Proof. The approach we will take is via the relations

$$
\begin{aligned}
& \lim _{t \rightarrow 0+} E_{a}\left\{\frac{y(t)-a}{t}\right\}=m(a), \\
& \lim _{t \rightarrow 0+} E_{a}\left\{\frac{[y(t)-a]^{2}}{t}\right\}=1, \text { and } \\
& \lim _{t \rightarrow 0+} E_{a}\left\{\frac{[y(t)-a]^{4}}{t}\right\}=0 .
\end{aligned}
$$

(" $E_{a}$ " is expectation with respect to the measure $P_{a}$ ).

These facts are almost immediate from (1) when $m$ is bounded, but to handle other cases we need to use (4), together with

$$
E\left\{\max _{\tau \leq t}|x(\tau)|^{k}\right\}=C_{k} t^{k / 2}, \quad k=1,2, \cdots,
$$

where " $E$ " means integral with respect to Wiener measure. (The last fact is easy, since the exact distribution is known from the "reflection principle".) Equation (9) is an immediate consequence of (4) and (10).

To derive (7) and (8) is now very easy. Writing

$$
y(t)-a=x(t)+t m(a)+\xi(t)
$$

where 


$$
\xi(t)=\int_{0}^{t}\{m(y(t))-m(a)\} d \tau,
$$

we will have (7) as soon as we show that $E(\xi(t))=o(t)$. But making use of (4) we have for small $t$

$$
|\xi(t)| \leq 2 K t\left(t|m(a)|+\max _{\tau \leq t}|x(\tau)|\right),
$$

and using (10) we have $E(|\xi(t)|)=o\left(t^{3 / 2}\right)$ and hence (7). To obtain (8) is equally simple; from (11) we have at once

$$
(y(t)-a)^{2}=x(t)^{2}+[\operatorname{tm}(a)+\xi(t)]^{2}+2 x(t)[\operatorname{tm}(a)+\xi(t)] .
$$

On taking expectations the first term gives the desired answer $t$, and it is easy using the facts above to see that the remaining terms are $o\left(t^{2}\right) ;(8)$ follows.

New suppose that $u$ is bounded and has a continuous second derivative. There is a well-known simple argument, based on the Taylor expansion of $u$ up to the quadratic term, which shows that for each a

$$
\lim _{t \rightarrow 0-} \frac{\left(\boldsymbol{T}_{t} u\right)(a)-u(a)}{t}=m(a) u^{\prime}(a)+\frac{1}{2} u^{\prime \prime}(a)
$$

we will not give the details. The only properties of the semi-group which come into play are (7), (8) and (9). If, moreover, $u \in C_{0}$, it is obvious that for $a= \pm \infty$ the limit in (12) exists and equals 0 , since by definition $\left(\boldsymbol{T}_{t} u\right)( \pm \infty)=u( \pm \infty)$, If finally $u$ is such that the right side of (12) is continuous at $\pm \infty$ (i.e. tends to 0 ), then it is known $^{2)}$ that the convergence must be uniform and so that $u$ must belong to the domain of the generator $\boldsymbol{A}$.

It only remains to see that the whole of the domain has been identified. Since, for large $\lambda$, the operator $\lambda-\boldsymbol{A}$ maps the domain of $\boldsymbol{A}$ onto $C_{0}$, it suffices to show that the operator in (6), with its "natural" domain described above, also has this property to establish that it coincides with $\boldsymbol{A}$. In other words, we need to be sure that the equation

2) This and other facts from semi-group theory used below are proved, for instance, in [8]. 


$$
\lambda u-m(x) u^{\prime}-\frac{1}{2} u^{\prime \prime}=u(x)
$$

has a solution $u$ in the natural domain of (6) for every $v \in C_{0}$. A more general result has however been proved by Feller $([6]$, Thm. 13. 1) and so the proof of Theorem 1 is complete.

\section{Generator of $\{z(t)\}$}

We now consider the effect of the second stage of the construction - the transformation $z(t)=f(y(t))$ - on the infinitesimal generator. For simplicity we always assume that $f$ maps the line onto itself. Then $\{z(t)\}$ gives rise to another semi-group $\left\{\boldsymbol{S}_{t}\right\}$ of operators on $C_{0}$; let $\boldsymbol{B}$ denote its generator. The point of departure is the simple observation that

$$
\left(\boldsymbol{S}_{t} v\right)(b)=\left\{\boldsymbol{T}_{t}(v \circ f)\right\}(a),
$$

where $v \circ f$ means the composed function $v(f(a))$ and $b=f(a)$. It follows that $v$ is in the domain of $\boldsymbol{B}$ if and only if $v o f$ is in that of $\boldsymbol{A}$, and when this holds

$$
(\boldsymbol{B} v)(b)=\{\boldsymbol{A}(v \circ f)\}(a) .
$$

We will now make, at some loss of generality, the assumption that $f^{\prime \prime}(a)$ exists and is continuous on $(-\infty,+\infty)$. It is then clear from the above discussion together with Theorem 1 that $\boldsymbol{B}$ has the form

$$
(\boldsymbol{B} v)(b)=\frac{1}{2} f^{\prime}(a)^{2} v^{\prime \prime}(b)+v^{\prime}(b)\left\{\frac{1}{2} f^{\prime \prime}(a)+m(a) f^{\prime}(a)\right\}
$$

when $v$ is in its domain ; this occurs exactly when $v^{\prime \prime}$ is continuous and the right side of (14) tends to 0 as $a \rightarrow \pm \infty$ (recall $b=f(a)$ ). Hence in this case $\boldsymbol{B}$ is a classical diffusion operator of the form

$$
(\boldsymbol{B} v)(b)=\frac{\alpha(b)^{2}}{2} v^{\prime \prime}(b)+\beta(b) v^{\prime}(b) ; \quad \alpha(b) \geq 0 .
$$

We will next give sufficient conditions iusuring that an operator (15) corresponds to a process obtained from our construction :

Theorem 2. There is a process $\{z(t)\}$, constructed by the method in section 1, which has (15) for its generator provided that 
$\alpha^{\prime \prime}$ and $\beta^{\prime}$ exist, that $\alpha>0$ and $\frac{1}{\alpha}$ is not integrable at either $\pm \infty$, and that the functions $\alpha \alpha^{\prime \prime}$ and $\beta^{\prime}-\beta \alpha^{\prime} / \alpha$ are bounded ${ }^{3)}$. If these conditions hold, the function $m$ and $f$ used in the construction are given by (17) and (16) below.

Proof. Comparing the generator which has been obtained in (14) for $\{z(t)\}$ with the general form (15), we see that if they agree it is necessary that $f^{\prime}(a)=\alpha(b)$. This yield

$$
f(a)=S^{-1}(b), \quad \text { where } \quad S(b)=\int_{0}^{b} \frac{d x}{\alpha(x)} .
$$

The assumption that $f^{\prime \prime}$ is continuous requires that $\alpha^{\prime}$ be continuous and the need for $f$ to map the whole line onto itself is met provided $\alpha(b)>0$ for each $b$ and $S( \pm \infty)= \pm \infty$.

Again comparing (14) and (15) we obtain

$$
m(a)=\frac{\beta(b)-\frac{1}{2} \alpha^{\prime}(b) \alpha(b)}{\alpha(b)} .
$$

In the construction of $\{y(t)\}$ it was assumed that $m$ satisfied a uniform Lipschitz condition; if such a condition holds for the function of (17) then the ultimate construction of a process $\{z(t)\}$ with generator (15) will be possible. It is, of course, enough that

$$
\left|\frac{d}{d a}\left(\frac{\beta(b)}{\alpha(b)}\right)\right| \leq M \quad \text { and } \quad\left|\frac{d}{d a} \alpha^{\prime}(b)\right| \leq M
$$

should hold, but using $\frac{d b}{d a}=f^{\prime}(a)=\alpha(b)$ these statements reduce to the hypotheses of the theorem, and so the proof is complete.

\section{An application}

The main theoretical advantage of the construction described above is that it simplifies the analysis of the sample functions of $\{z(t)\}$ in terms of the Wiener paths. For instance, it is well known that a Wiener path is nowhere differentiable, with probability 1 ,

3) The proof will show that if $\alpha$ is bounded, the other boundedness conditions can be replaced by the assumption that $\alpha^{\prime}$ and $\beta / \alpha$ satisfy a Lipschitz condition of order 1 , and the existence of $\alpha^{\prime \prime}$ and $\beta^{\prime}$ everywhere is not needed. 
and in fact it nowhere satisfies a local Hölder condition of order greater than $1 / 2$. From (1), it is then immediate that the paths of $\{y(t)\}$ have the same properties, and provided $f$ has a continuous, positive derivative the same things hold for $\{z(t)\}$. In particular, processes with a generator satisfying the assumptions of Theorem 2 all have paths which are (a.s.) nowhere differentiable. In much the same way other properties of Brownian motion paths can be carried over to a large class of diffusions. Some of these will be mentioned explicitly in the next theorem, although no attempt was made to provide an exhaustive list.

Theorem 3. Let $\{z(t)\}$ be any diffusion constructed by the method of section 1, where the function $f$ has a continuous, positive derivative. Then

(i) With probabiliey $1, z(t)+$ ct has no points of increase or decrease for any value of $c$.

(ii) With probability 1, z(t) has an upper (lower) derivative equal to $+\infty(-\infty)$ everywhere ${ }^{4}$.

(iii) With probability 1 the equantity

$$
\lim _{\delta \rightarrow 0+} \sup _{\substack{\mid t_{1}-t_{2} \leq \delta \leq \\ t_{1}, t_{2} \leq T}} \frac{\left|z\left(t_{1}\right)-z\left(t_{2}\right)\right|}{\left|t_{1}-t_{2}\right|^{1 / 2}\left|\log \left(t_{1}-t_{2}\right)\right|^{1 / 2}}
$$

is finite; it is bounded provided $f^{\prime}$ is bounded.

(iv) For each a, with probability 1

$$
\left.\limsup _{t \rightarrow 0+} \frac{z(t)-b}{\mid 2 t} \frac{\left.\log ^{\prime} \log t\right|^{1 / 2}}{\mid}=f^{\prime}\left(f^{-1}\right)(b)\right) .
$$

Proof. Each of these results can easily be deduced from known properties of the Wiener paths, together with our representation of the diffusion $\{z(t)\}$. For instance, it was proved in [4] that $x(t)+c t$ has no points of increase or decrease for any $c$, for almost all Wiener paths $x(t)$. But if $x(t)$ and $y(t)$ satisfy (1) it is clear that the existence of a point of increase for $y(t)+c t$ implies one for $x(t)+c^{\prime \prime} t$ for all large enough $c^{\prime \prime}$, so that $\{y(t)+c t\}$ has

4) Here "upper derivative" means the (two sided) lim. sup. of the difference quotients. 
a.s. no points of increase (or decrease). Since $f$ is strictly monotonic, it is immediate that $z(t)=f(y(t))$ can have no point of increase or decrease; the same result for $z(t)+c t$ follows using the assumed differentiability of $f$. This proves (i), and (ii) is an immediate corollary. The Hölder continuity asserted in (iii) follows from the analogous result for Brownian motion which is proved in [1]; the local law of the interated logarithm (iv) is also an immediate consequence of the law in the Brownian case, which is well known.

\section{Concluding remarks}

The class of processes described in Theorem 1 is of course a restricted one; the assumptions made there are somewhat similar to those made by Feller in his early work on diffusion [6]. The approach we have taken could be generalized, for it is not always necessary in the first stage that $m$ be a Lipschitz function (see $[10])$ nor, in the second stage, that $f^{\prime}$ exist everywhere. It would be of some interest to see how large a class of processes can be obtained by our method, although the only advantages which it offers over other, more general method-those of simplicity and the results in Theorem 3-might well be lost in the process.

It is easy to incorporate absorbing barriers into the diffusions we have constructed. This can be done for the process $\{y(t)\}$ by changing the mapping $M_{a}$ to provide that, for each $x(t)$, the corresponding $y$ (starting inside the desired open state interval) proceeds as before until it first touches a barrier and then remains constant thereafter. The second step of the construction, of course, merely changes the position of the boundaries. Reflecting barriers are also readily provided, as $\mathrm{H}$. McKean pointed out to me. To illustrate the method, suppose a barrier at 0 is desired. The function $m$ is originally given on the positive half line; we extend it as an even function. Using the extended $m$, a process $\{y(t)\}$ on the whole line is constructed as in section $1 ;\{|y(t)|\}$ then provides the desired reflecting barrier process. Two barriers can be managed in much the same way, and again the second stage of the con- 
struction introduces no difficulty. In both the absorbing and reflecting cases the analysis of the infinitesimal generator proceeds with only minor changes from the discussion in section 2 and 3 , and shows that functions in the domain of $\boldsymbol{A}$ must satisfy appropriate classical boundary conditions.

Finally we mention the possibilities of taking the same approach to Markov processes with discontinuous paths. This has been done using the method of stochastic integral equations by K. Itô [9]; a special case was suggested by Doob in [2]. If $x(t)$ is any bounded measurable function, the usual existence proof still shows that (1) has a unique solution, and so it can be shown that processes $\{y(t)\}$ (and hence also $\{z(t)\})$ can be constructed from a process $\{x(t)\}$ with "reasonable" discontinuous paths by following the steps of our construction for diffusions. It is then not hard to prove that if $\{x(t)\}$ has stationary independent increments, the resulting process will be Markovian with stationary transition probabilities. This strongly suggests the possibility of generalizing a.s. results on the paths of differential processes to a wider class in a manner analogous to Theorem 3. We shall not pursue this further at present, however.

\section{REFERENCES}

[1] Z. Ciesielski, "Hölder conditions for realizations of Gaussian processes", Trans. Amer. Math. Soc. 99 (1961), pp. 403-413.

[2] J. L. Doob, "The Brownian movement and stochastic equations," Ann. of Math. 43 (1942), pp. 351-369.

[3] J. L. Doob, Stochastic Processes, New York (Wiley), 1953.

[4] A. Dvoretzky, P. Erdös, and S. Kakutani, "Nonincrease everywhere of the Brownian motion process," Proc. Fourth Berkeley Symp. vol. 2, pp. 102-116, University of California 1961.

[5] E. B. Dynkin and A. A. Yushkevich, "Strong Markov processes," Theory of Prob. and Applications, (1956), pp. 134-139. (Translation from Russian.)

[6] W. Feller, "Zur theorie der stochastischen Prozesse," Math. Ann. 113 (1936), pp. 113-160.

[7] W. Feller, "The parabolic differential equations and the associated semi-groups of transformations," Ann. of Math. (1952), pp. 468-519.

[8] K. Ito, Stochastic Processes, Tokyo, 1957. (Mimeographed translation by Yuji Ito, 1961.)

[9] K. Ito, “On stochastic differential equations," Mem. Amer. Math. Soc. 4 (1951).

[10] H. P. McKean, Jr., "The Bessel motion and a singular integral equation," Mem. Coll. Science Kyoto Univ., A, vol. XXXIII (1960), pp. 317-322. 